\title{
Big DATA AND BUSINESS INTELLIGENCE: RESEARCH AND CHALlengeS IN TELECOM INDUSTRY
}

\author{
Ivan Krasic, Stipe Celar \& Zeljko Seremet
}
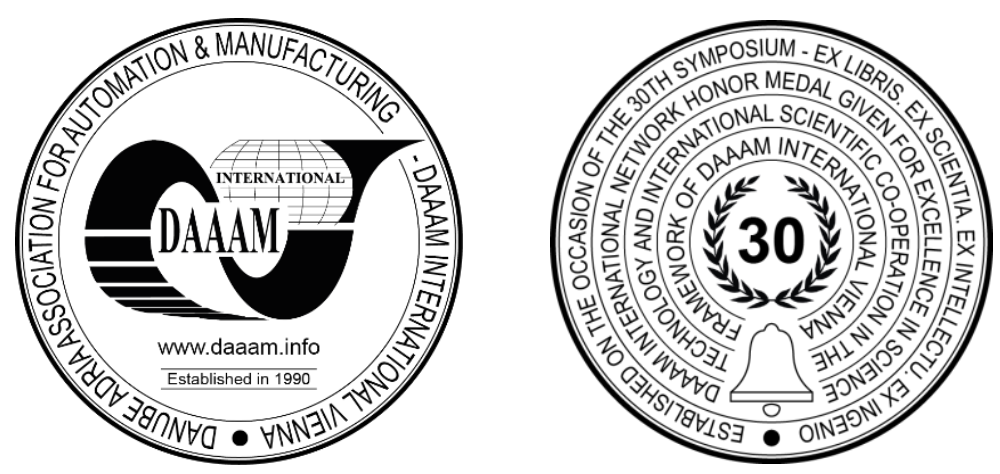

This Publication has to be referred as: Krasic, I[van]; Celar, S[tipe] \& Seremet, Z[eljko] (2021). Big Data and Business Intelligence: Research and Challenges in Telecom Industry, Proceedings of the 32nd DAAAM International Symposium, pp.0339-0348, B. Katalinic (Ed.), Published by DAAAM International, ISBN 978-3-902734-33-4, ISSN 1726-9679, Vienna, Austria

DOI: $10.2507 / 32$ nd.daaam.proceedings.050

\begin{abstract}
This paper focuses on the Big Data (BD) and Business Intelligence (BI) application in the telecom domain and the benefits it offers. Telecom companies themselves are the producers of big data on daily basis - structured and unstructured data formats from many sources. Already some level of Business Intelligences is implemented and in-use for supporting telecom daily business. We provide literature review of BD and BI state-of-art in telecom domain, example of real world use cases of BD and BI analytic, platforms, frameworks, practices and the related challenges of the further development and research trends.
\end{abstract}

Keywords: big data; business intelligence; telecom; churn; literature review.

\section{Introduction}

The rapid development of telecommunications and other large systems over the last decades has resulted in increased system complexity and data itself. Telecom operators striving to analyse large volumes of data in order to support their core business and operations. Solution was to deploy sustainable storage data systems - which differs from operating/transactional systems of historical, unaltered and multidimensional organized data that are intuitive and adapted to thinking and human inference and analysis. Today, in highly competitive market, rising era of big data (BD) is characterization of all operatory whole around world. In addition to telecom changes, proliferation of other systems directly related to telecom like Internet, Internet of Things, and Cloud Computing have led to the explosive growth of data in almost every industry and business area, affecting telecom operators too.

This paper provides a short overview on how telecom operators have been striving to analyse large volumes of data in past. Thus, we explain big data paradigm with relation to business intelligence (BI) process. We then review state-ofart in BD and BI in telecom domain. Finally, we provide example of real world use cases of BD and BI analytic, platforms, frameworks, practices and the related challenges of the further development and research trends. 


\section{Business intelligence}

Business intelligence, even used in more decades, is not a well-defined term, depends on who is defining. Some authors as [1] define BI as "data reporting and visualization, some consider as business performance management. Database vendors emphasize data extraction, transformation and integration features. Analytics vendors highlight statistical analysis and data mining". Also it can be defined as system that can be used for making decision from information based on available data - process which covers: integration and collection of data sources, storage and information database, management of knowledge with the use of different systematic and analytical tools in presenting information to the organization's decision makers and stakeholders. They should take an action (decision) based on BI environment back to origin systems (OSS, Network etc.) or send it as a input of toll based on artificial intelligence, releasing action too. Typical BI architecture consist of following components, connected by unidirectional communication flow between different components (Figure 2):

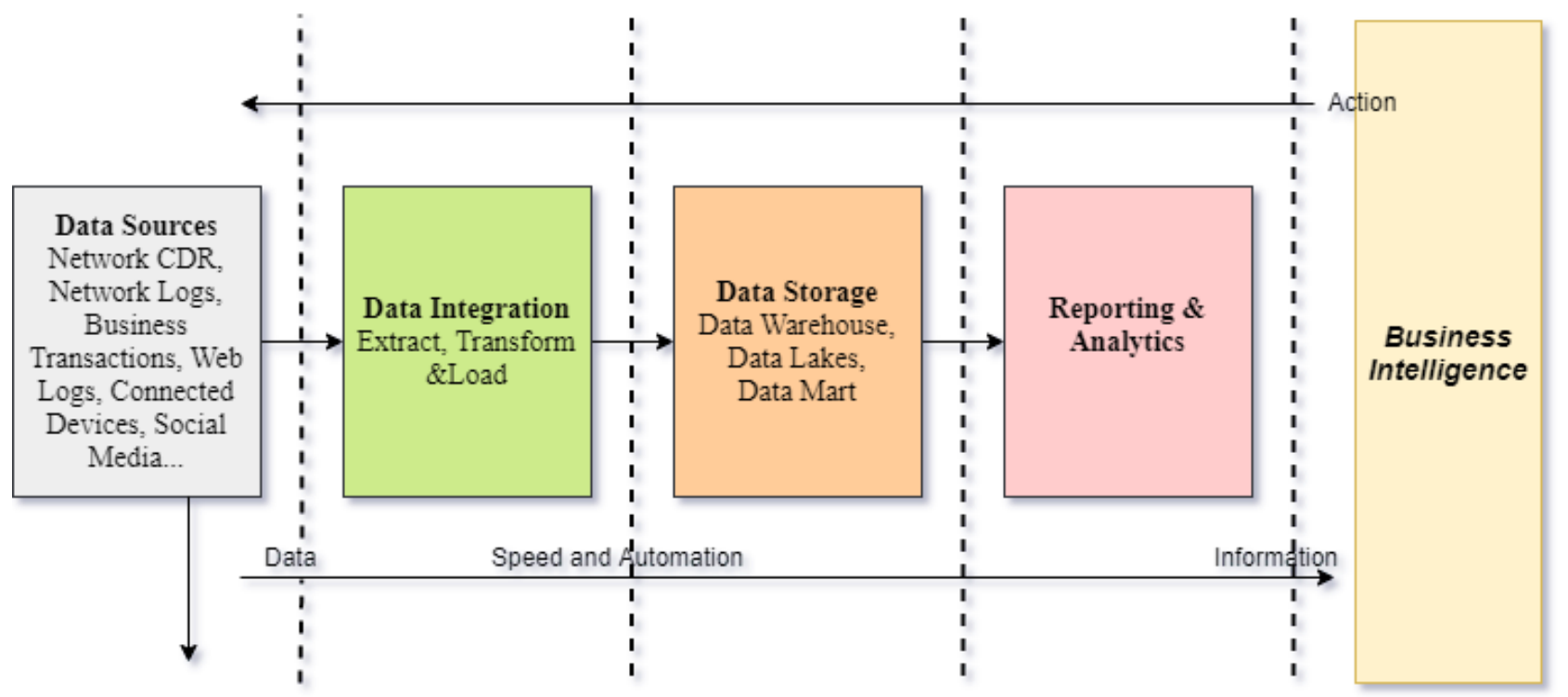

Fig. 1. Typical BI architecture

Typical BI architecture (presented in Figure 1) consist of the following layers:

- Data Sources - include all data from organization's systems, external sources too, structured on unstructured, many different formats, with different nature in sense of characteristics (volume, velocity,...).

- Data Integration - provide extraction, transformation and integration of data via appropriate tools, from many sources, automated tasks with remark of speed and automation level.

- Data Storage - layer responsible for data storage, on premise or cloud, organized in classic way (relational database management system) or specialized databases that provide control over selected activities and selected business process (data warehouse, data mart, data layers etc.).

- Reporting \& Analytics - subset of different software tools exploiting stored data, in order to make different reports, analyse specific or ordinary business needs, or even make proactive tasks. In addition, this layer provide specialized different $\mathrm{W}$ analyses (Why, When, Who, What, What If...).

This simplified architecture present classic and typical BI framework deployed in many telecommunication companies today. Typical BI deployment and framework are widely accepted and are de-facto standard for business and corporate analytics and decision support systems (DSS). On the other side this deployment scenario is not appropriate for upcoming challenges in rapid growth of data volume, velocity and variety.

\section{Big data}

The term "big data" has long history and there is no single standard definition. Some authors finding origins in academic and non-academic community from 1990-th, even earlier, but truly come of age in 2008. - report "Big Data Computing: Creating revolutionary breakthroughs in commerce, science, and society" from Computing Community Consortium and in age of 2013 - when Oxford English Dictionary introduced the term "Big Data" for the first time in its dictionary [2]. In the last decade big data research has significantly increased (Figure 1). 
google trends from 2004.-2020.

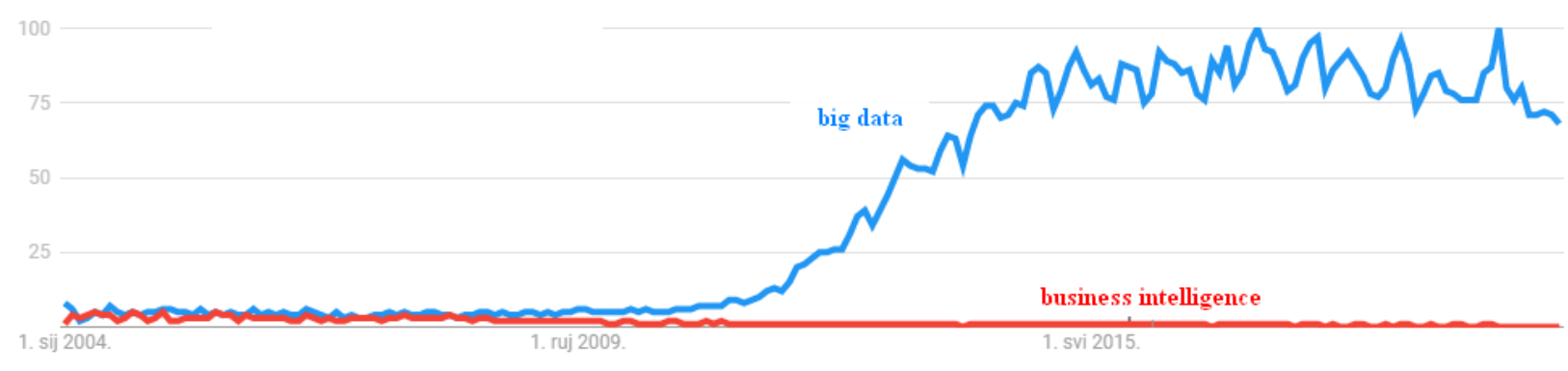

Fig. 2. Google trends for "big data" and "business intelligence” (2004-2020)

Big data trends have been enabled by advances in computing technologies, which facilitated the sudden explosion of data from various sources such: Internet and Web, social medias, sensors etc. The large amount of data emergence application of a data-driven paradigm to take advantage of the newly available computing technologies. Big data technologies materialized the data-driven paradigm, making it increasingly sophisticated and useful [3].

Today, Big Data (big data or BD) is a comprehensive term that primarily refers to the sources of large amounts of data and the infrastructure that supports their storage. Data access requires new tools and technologies (framework) for optimized access. Numerous languages have been developed for the analysis of new sources, real time solutions, security concepts, etc. Big data includes technology that serves to collect process and complex analyse a large amount of data. Data are diverse, structured and unstructured, they are generated and arrive at high speed at different intervals (asynchronous or real time).

Big data is often characterized by different V's, according to leading letter " $\mathrm{V}$ " in characterize - originally $3 \mathrm{~V}$, then some authors proposed $4 \mathrm{~V}, 5 \mathrm{~V}, 6 \mathrm{~V}, 7 \mathrm{~V} \ldots$ :

\begin{tabular}{|c|c|c|}
\hline Vs & Characheristic & Description \\
\hline \multirow[t]{3}{*}{$3 \mathbf{3 V}$} & Volume & Scaling - data volume is increasing exponentially, quantity measure \\
\hline & Variety & Complexity - various formats, types, and structures, \\
\hline & Velocity & $\begin{array}{l}\text { Speed - data is generated fast and need to be processed fast (real time } \\
\text { application, etc...) }\end{array}$ \\
\hline $4 \mathrm{~V}$ & Veracity & $\begin{array}{l}\text { Confidence or Completeness or trust in the data. This is similar to, but not the } \\
\text { same as, validity or volatility. }\end{array}$ \\
\hline $5 \mathrm{~V}$ & Value & $\begin{array}{l}\text { Need to derive business value from the data - like customers data, targeting } \\
\text { them, optimizing processes or improving machine or business performance. }\end{array}$ \\
\hline $6 \mathrm{~V}$ & Validity & Refers to how accurate and correct the data is for its further use. \\
\hline $7 \mathrm{~V}$ & Volatility & Refers to how long is data valid and how long should it be stored. \\
\hline \multirow[t]{3}{*}{$\begin{array}{l}10 \mathrm{~V} \\
\cdots\end{array}$} & Variability & $\begin{array}{l}\text { Refers to number of inconsistencies in the data and inconsistent speed of data } \\
\text { loading into database or any storage layer. }\end{array}$ \\
\hline & Vulnerability & Big data requires new security concerns. \\
\hline & Visualization & Characteristic of big data is how solve new visualization chellenges. \\
\hline
\end{tabular}

Table 1. List of V's of Big Data

Concerning that big data access is primary data-driven based, similar as in past, where storing data is integrated from one or more sources (operating systems etc.), building any data storage is a complex and thankless job. It follows the steps like other information systems but with one difference that the impact of the project warehouse is important and implies specific knowledge about certain problems and other skills. The projector warehouse was responsible for the model warehouse and later (un)/successfully used it. The choice of measures / measurement data to be analysed and contained in the multidimensional model is the role of the designer.

Rising of big data technologies other roles should be introduced and developed, leaving the past known human roles and applied technologies. Comprehensive review study about BI and BD research evolution and interaction and can be found in [4]. 


\section{Literature review search and analysis}

In this paper we concentrates on searching in scientific databases - rather than in specific books or technical reports.

\subsection{Literature review search process}

This paper has selected next databases to perform the search process as follows:

1. Science Direct (www.sciencedirect.com)

2. Scopus ( https://www.scopus.com/home.uri )

3. Google Scholar (www.scholar.google.com)

4. IEEE Explore (https://ieeexplore.ieee.org/)

5. ACM Digital Library (https://dl.acm.org/)

6. Springer (https://www.springer.com/gp )

The databases were chosen as they offer the most important and highest impact full-text journals and conference proceedings, covering the requested fields (big data, telecom/(munication)). For this work paper we found and include scientific papers from Science Direct, Scopus and other listed above sources. In order to define a search string, we experimented with a variety of combinations of keywords to test synonyms used in literature (telecom or telco or telecommunication(s)). After few iterations we finally ended up with the following search string:

\section{“Big data” AND ("telecom” OR “telecommunication”)}

Search is done by simple keywords in article title, abstract or content, regardless of how specific search engines works at by itself. We started our search all well-known digital libraries, namely Science Direct, Scopus, IEEE Explorer, ACM, Springer, and Google Scholar. This whole process, including initial and result set, consist of following steps (Figure 3).

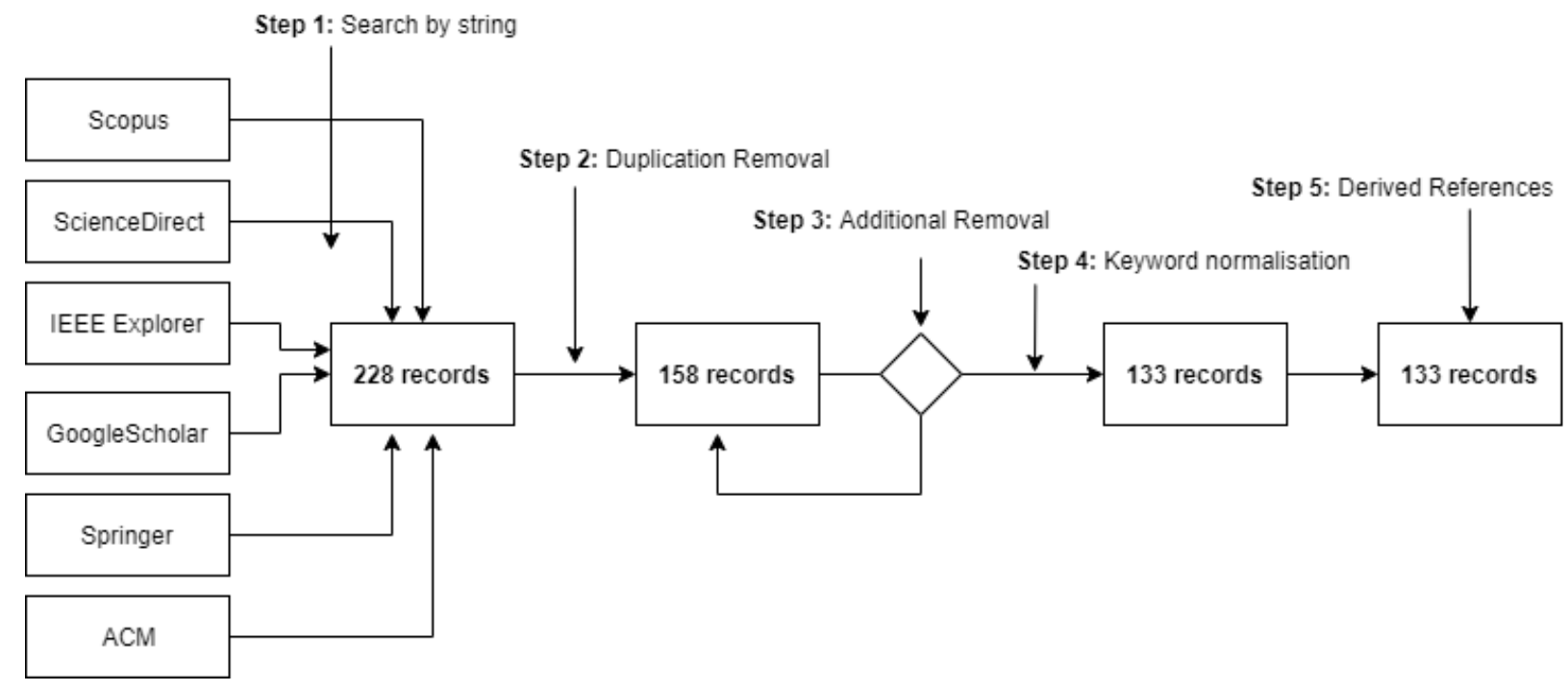

Fig. 3. Literature search process

We followed these procedure steps:

Step 1 Search digital libraries: search by keywords;

Step 2 Duplication Removal: After consideration of the 228 publications we found that 30,7\% papers were duplicate. After discarding duplicates, we were left with 158 articles;

Step 3 Additional Removal: this step is done by following simple inclusion/exclusion criteria: "The language of the literature is English", "Author keywords must be defined", "Paper has to be either journal article, conference paper of book chapter and publisher".

Step 4 Keywords normalisation: update keywords, from plural to singular (\{telecommunications $\}$, $\{$ telecommunication $\} \ldots$ ), abbreviations ( $\{$ call detail record $(s),\{C D R\},\{c d r\} \ldots\}$

Step 5 Derived references: final reference list is available for further processing in different formats $\left({ }^{*}\right.$.ris, *.bib...)

The most of them are journal articles, with one or more authors, order by autonomous search engine ranking (best match etc...). Furthermore, next step in analysis is using Mendelay tool, reference manager desktop software, for constructing library of selected articles. Exported reference list is available in BibTeX (*.bib) or Research Information System (*.ris) document format. 


\subsection{Literature review analysis}

In this review, we identify and analyse the selected papers in order to a knowledge base that aims to summarize more clearly the key aspects of big data and analytics application in the telecom industry. This should bring values to both academia and practice, by providing both insights into what has already been studied with respect to the telecom development trends and a motivation for future research. Figure 4 indicates the distribution of selected papers by type notice that journal articles has the higher selected papers per type among conference proceeding and book chapter.

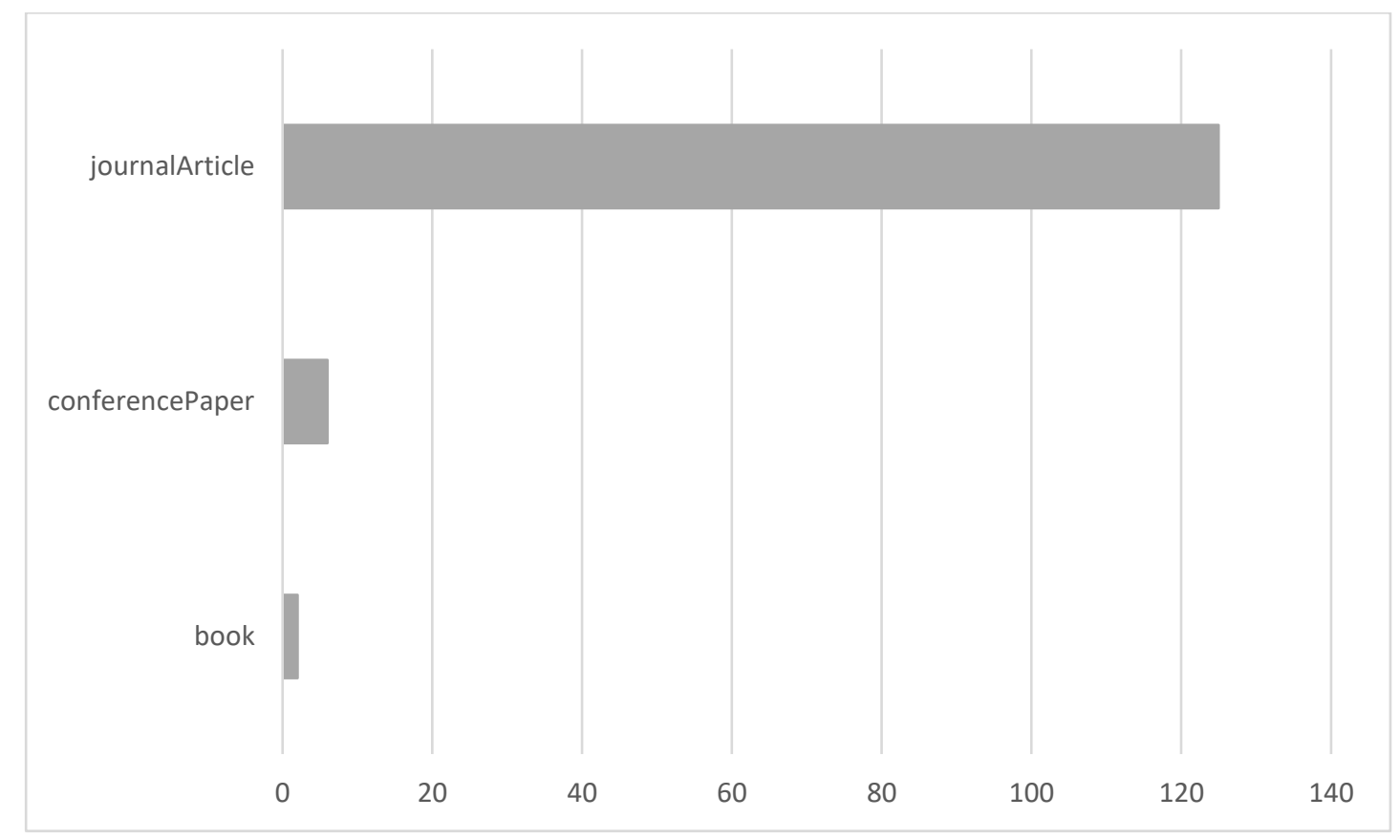

Fig. 4. Distribution of papers by type

Figure 5 shows distribution of papers by year in study. Regardless of the common perception that the "big data" started in the late 2000s when a dozen number of most prominent technology companies developed architectures to handle large-scale data (e.g., MapReduce by Google in 2004) "big data" is still a new term to the public and to academia. Joining the term "Telecom" to search we can detect increase in the number of scientific articles after 2015.

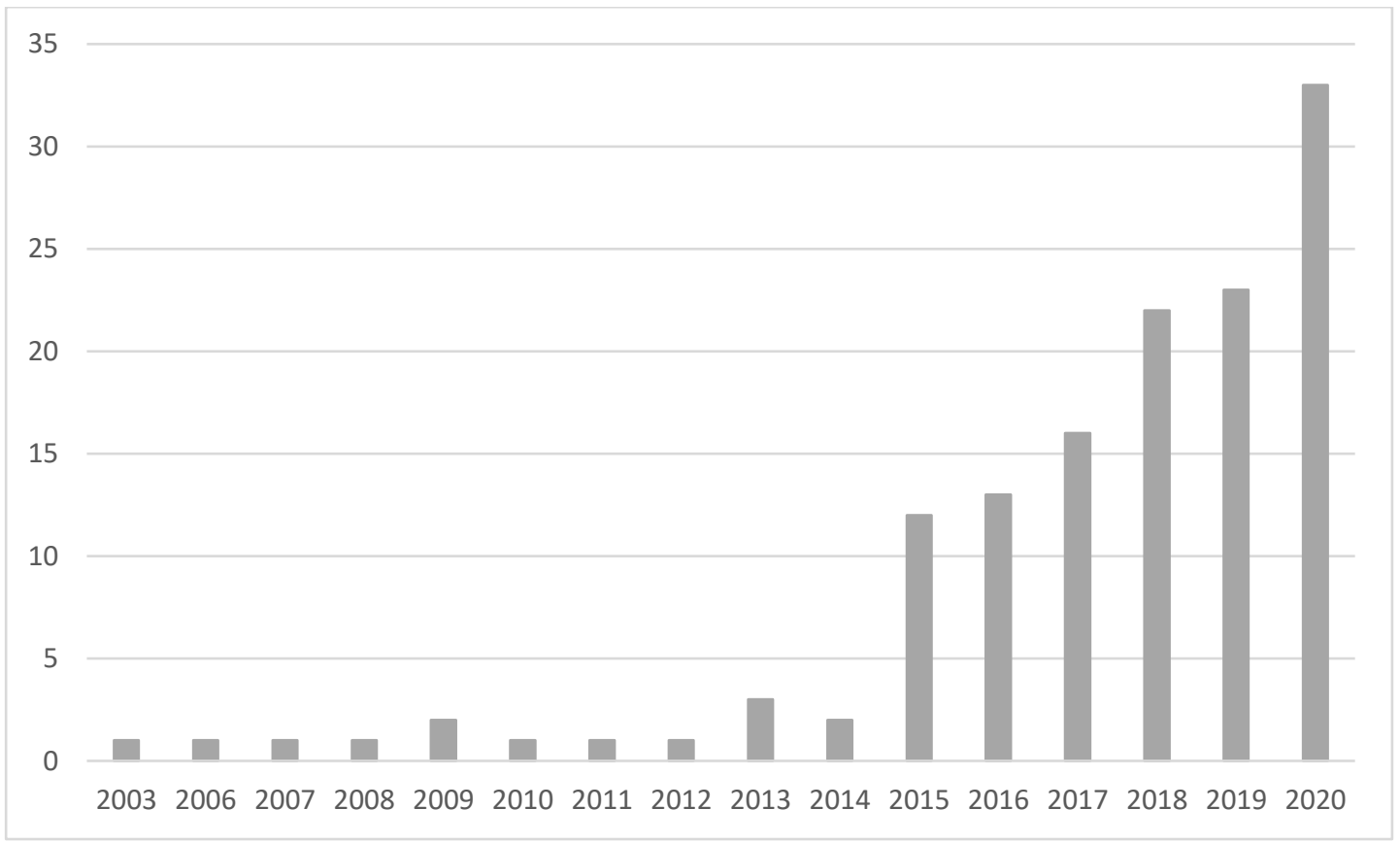

Fig. 5. Distribution of selected papers by year of publishing 
All of selected articles are published from year 2003, showing relatively weak interest up to 2015, excluding years 2009 and 2013. Thus, the number of publication is extremely increased in year 2015 and growing up to 2020, showing more research interest. Therefore, we are expected that years 2020- 2021 can reach higher than past few years, since this article list and review is based on export in May 2021.

\section{Results analysis}

In this section, we quantitatively and qualitatively analyse selected papers. The results of our work include the discovery of interactions in application of big data in telecom domain, in order to provide recommendations for all stakeholders (e.g., academic researchers, policy-makers, entrepreneurs...).

For this analysis we use tool VOSViewer for constructing and visualizing bibliometric networks. Network visualisation of keywords (by authors) is illustrated in Figure 3. The size of each circle corresponds to the proportion of keyword occurrences from picked papers.

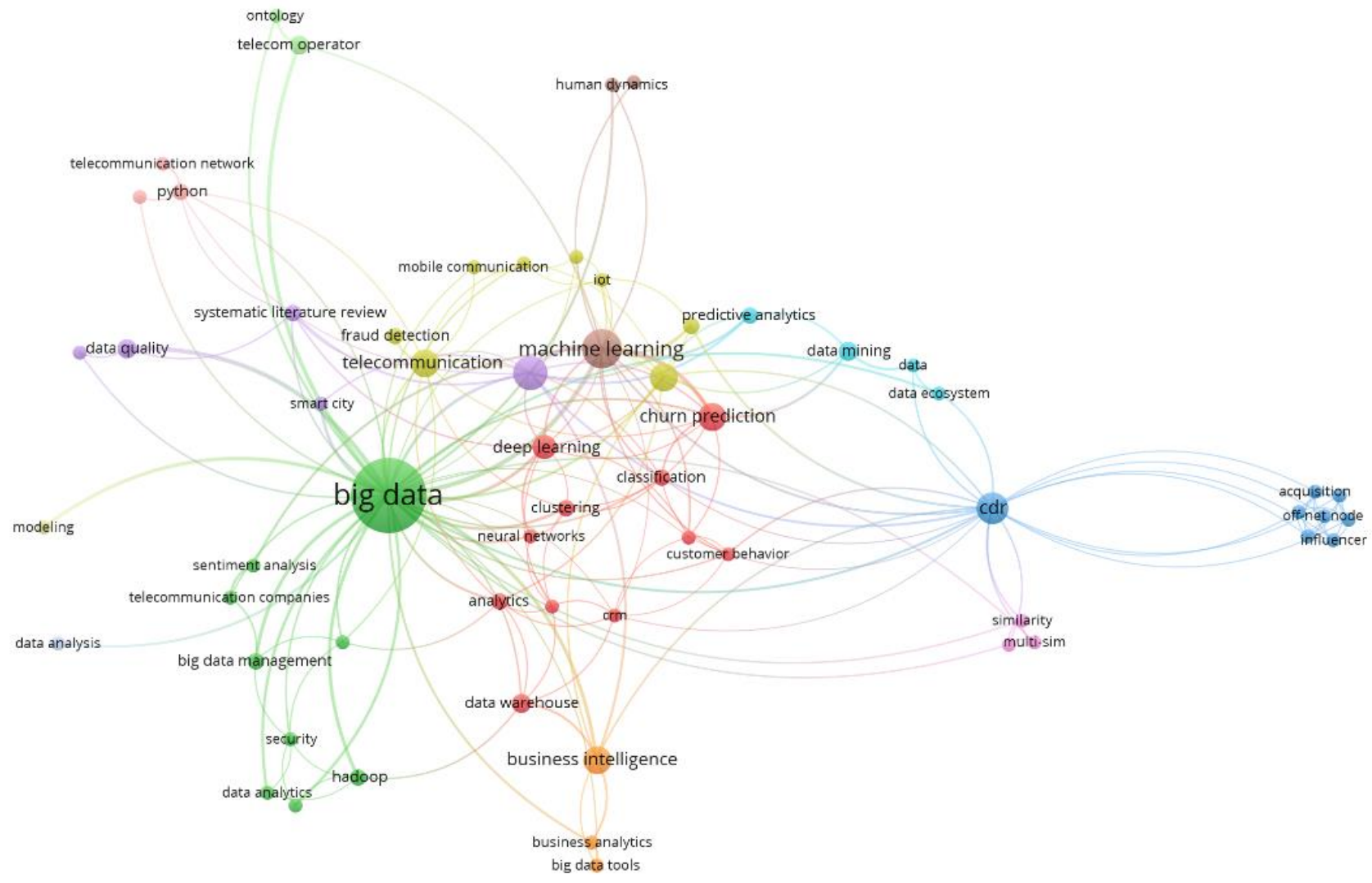

Fig. 6. Network visualization of keywords - word cloud of associated key words

For graph visualisation following values are exported:

- Total of different keywords (472)

- Minimum number of keyword occurrence (2) - exclude keywords with only one occurrence)

- Remaining keywords (62)

Decreasing minimum number of keyword occurrences can fit more specific use cases, maybe more interesting for telecom too but in this paper we start with default values. We can note relatively diversity of exported keywords,

Furthermore, the related keywords are grouped into different clusters categories and coloured with same colour (total number of clusters is 13). Following clusters are clearly detected in Figure 6, i.e., cloud computing (with MapReduce, Hadoop, and related techniques), machine learning (with a broad range of artificial intelligence techniques and applications), CDR (Call Detail Record), business intelligence and churn prediction term. Generally, there were no unexpected terms, all terms and their synonyms can be found on the other lists and are well known research topics. Looking into clusters distribution defined by VOS (13 in total) we can observe larger 5 clusters. Figure 5 displays the first cluster. 


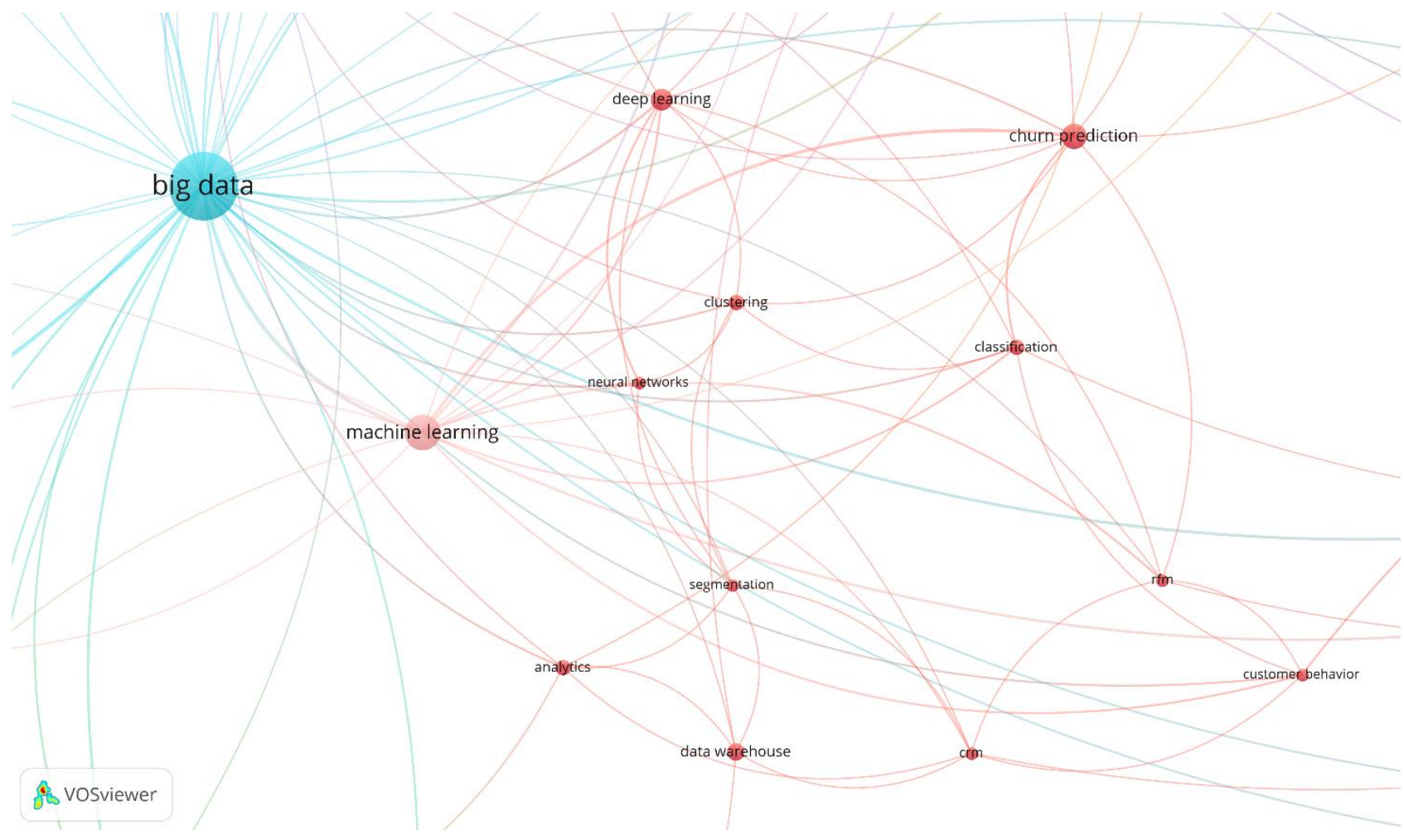

Fig. 7. Cluster_1 with prominent keyword categories big data and customer analysis

In above cluster we can see that big data is in strong connection with machine learning methods (clustering, deep learning, classification,...) in order to provide better way to customer related issues (churn prediction, customer related management,...).

The following cluster is shown in Figure 8.

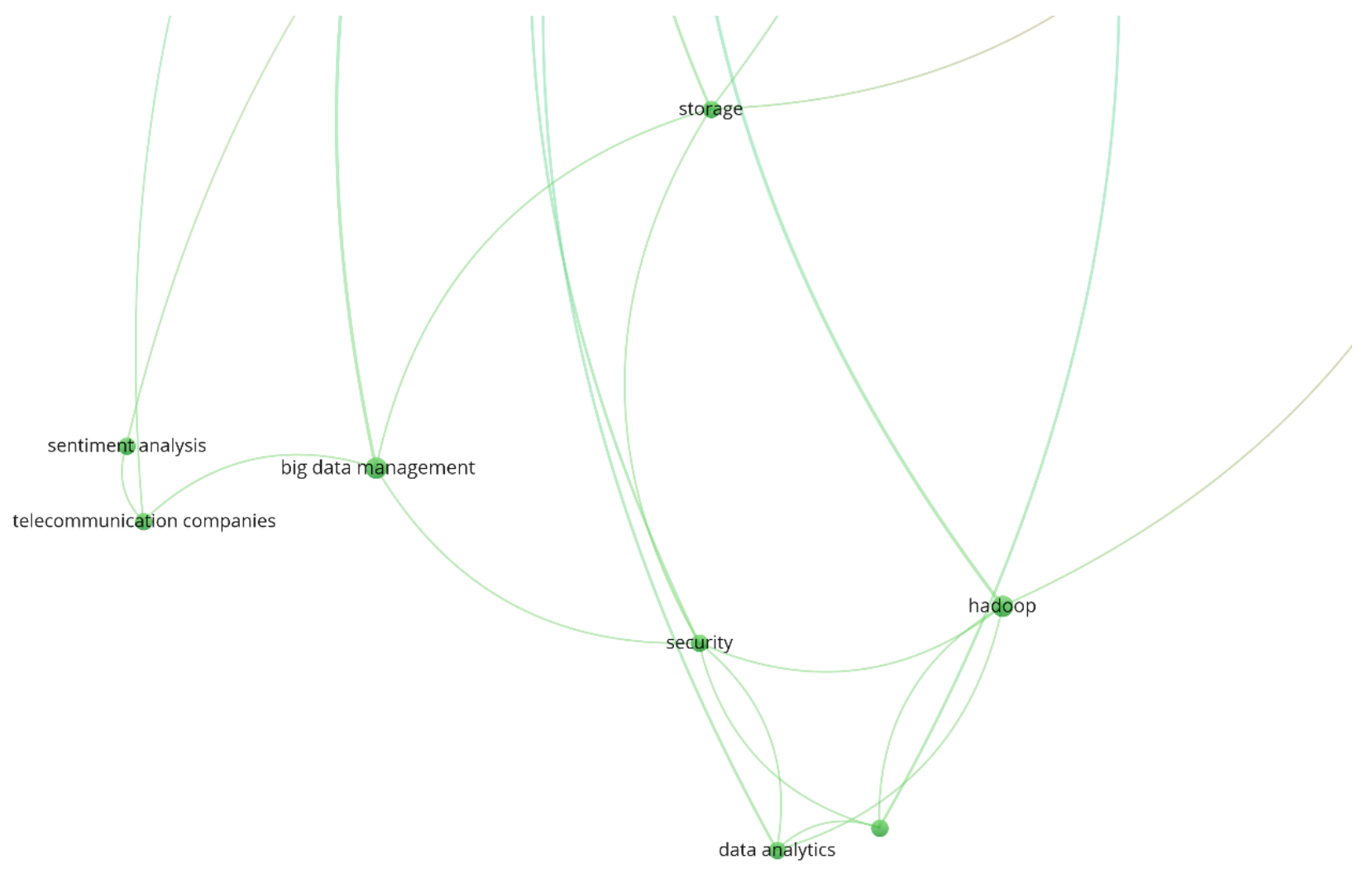

Fig. 8. Cluster_2 with prominent keyword categories big data and technology issues 
Cluster_2 is related to technology issues. For instance, it includes issues related to data storage, collection, analytics, processing and integration infrastructure (Hadoop). For example, sentimental analysis - collecting and analysing pure textual data in order to defining positive or negative reactions are also key research issues.

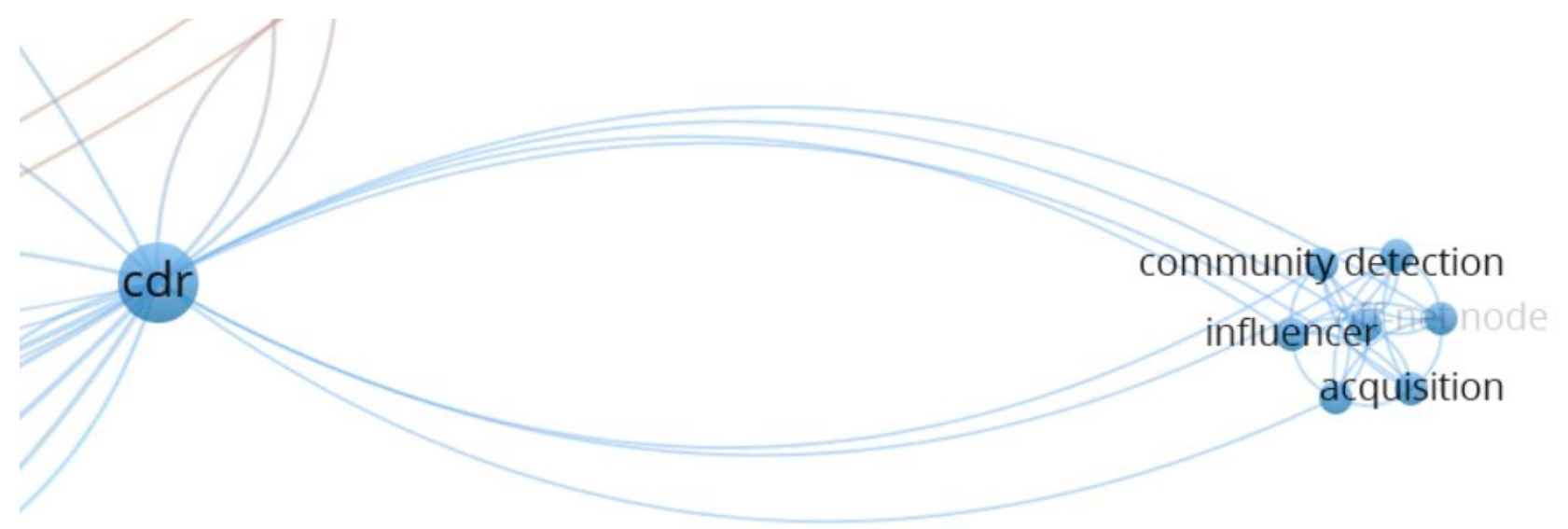

Fig. 9. Cluster_3 - CDR related analysis and research

In telecom industry, all data are stored in CDR files (Call Detail Records) and can be classified into three main groups: call detail data (related to communication between subscribers or to provider, calls, messages, data session), network $\underline{\text { data }}$ (all data from network systems and networks) and customer data (includes information about the personal data of customer such as name, address details, subscription type, payment history and other customer attributes).

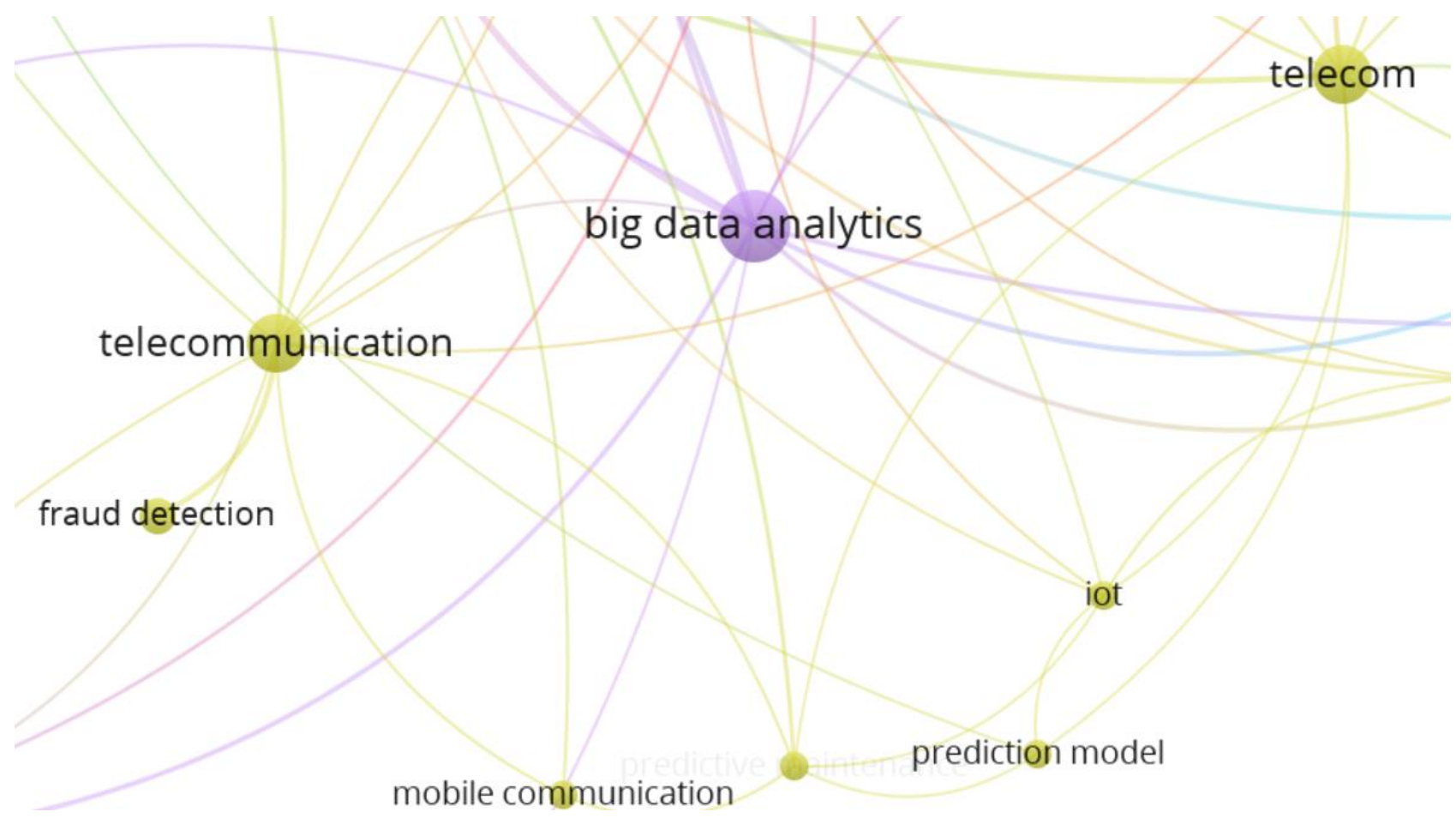

Fig. 10. Cluster_4 - Cluster related to specific telecom application

This cluster (Figure 10) is related to papers and research related to three specific areas: (1) specific telecom application like fraud detection and mobile communication, (2) newer trends like 5G and IoT (Internet of Things), and (3) network operation like predictive maintenance and prediction model (traffic), mainly in OSS domain (Operations Support System) 


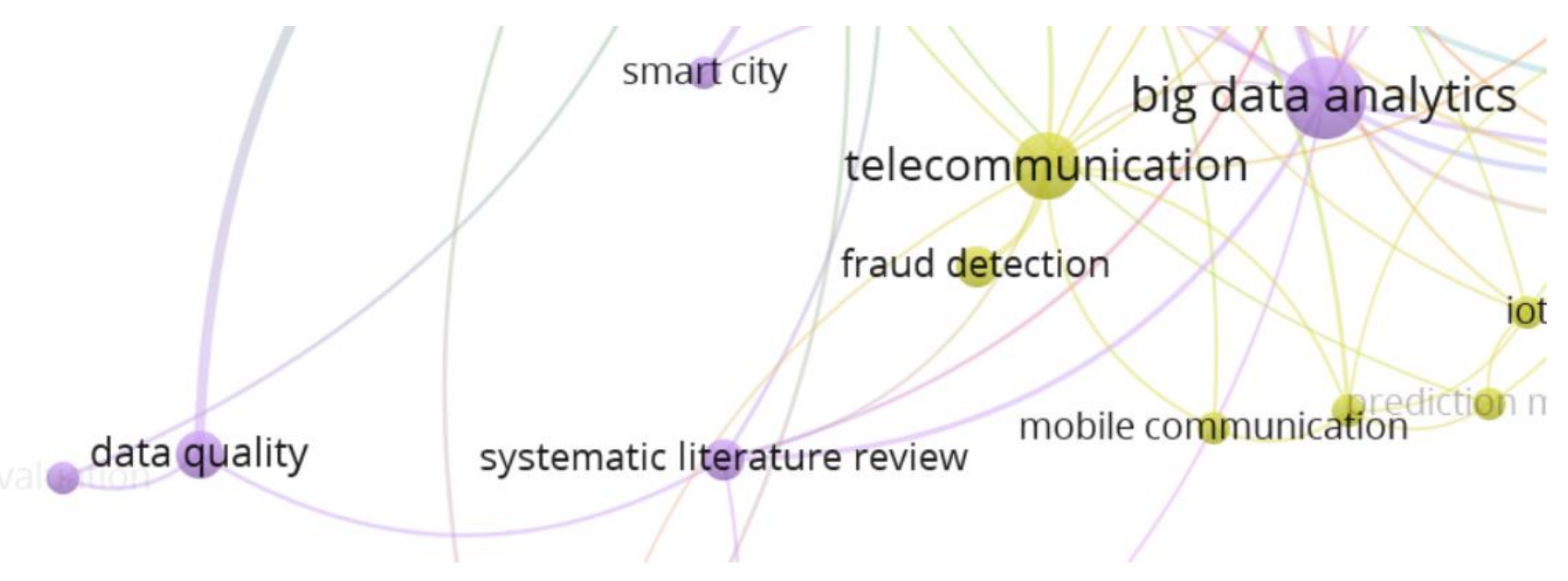

Fig. 11. Cluster_5 - Cluster related to big data analytics

This cluster (Figure 11, purple lines) is related to big data analytics in general, highlighting the topics related to data quality issues. In telecom operators multiple and variety data sources leads to data quality problems. Data quality in telecommunication systems has been studied from various perspectives, related to customer and network data.

Other clusters have smaller number of items $(<5)$ with larger diversity, and can observe them like more specific research interest, out of this paper.

\section{Big data challenges and research motivation}

According to presented correlation between research interests we can propose following challenges in telecom domain:

1. Challenges related to network services and management

2. Challenges related to customer acquisition and retention based

3. Challenges related to security issues

4. Challenges related to big data technology and management

\subsection{Challenges related to network services and management}

We can note several interesting research topics related to big data and application of machine learning methods to propose novel predictive maintenance frameworks modelling and implementation, reducing human actions and better error prevention. In case of service management, big data analytics can improve quality of service indicators (QoS KPI), provide more independent end to end service provision and management, also like shortening time to market in case of introducing new services and related products.

One more interesting research area is Robotic Process Automation (RPA) adopting in big data for automating telecom network management. Legacy telecom OSS and BSS are inadequate for the needs of the modern service providers because they were primarily designed to assist the humans in executing the processes that ran the business. To support the modern telecom needs, these systems need stronger automation and orchestration to operate the business quickly and autonomously under the control and oversight of people. This leads to roadmap for ultimately automation of all processes that run the telco business and management of the network.

\subsection{Challenges related to customer churn, acquisition and retention}

This group of challenges are well known topics like customer churn (unexpected leaving of operator), improving customer retention rates (customers who are retained over a specific time period) and better customer experience. This research topics are well none in past decades, data mining and business intelligence techniques are mainly applied to this issues. Introducing big data concepts and stronger machine learning methods can produce significant research contribution in future. According to [5], big data approach is applied for telecom churn prediction instead of use of data warehouse and data mining techniques, due to challenges of data volume, processing power and capacity management. Same authors quote that "acquiring a new customer costs six times higher than the cost of retaining the customer likely to churn". This indicates to direct conclusion that cost reduction and profit growth are well reasons to stronger research and real use case applications in this field. 


\subsection{Challenges related to security issues}

We can note several terms as Fraud detection (detection of fraud activities), Fraud Prevention (active and real time system for prevention of fraud, proactive approach), and other security issues that are related to data protection and privacy, as standard big data research problem. Telecom providers care many private and protected customer information like patterns of social behaviour, communication needs, location based data etc. From one hand this data can be very useful to take into account for providing future better customer experience, personalized service and products, but on other hand this requires very high level of security and privacy protection. Concerning security and privacy issues in big data environments for further research systematic literate review can be fine starting point [6]. Telecom domain fit all conditions to standard security and privacy problems research.

\subsection{Challenges related to big data technology and management}

This topics are already in research's focus for years. The technology dimension of big data includes issues related to data collection, storage, analytics, integration infrastructure and architecture. Finding best and appropriate infrastructure for telecom domain reflects the huge research interests, for example the cost-benefit assessment. The complexity of big data processing is one of the fastest growing technology challenges. Many tools and platforms are available for choice: open source or commercial, batch or stream or hybrid processing tools. We recommend further reading in [7] and [8].

\subsection{Other research challenges}

In this group web can place some research topics who are applicable for any other research group but also very interesting by itself. First of them is Data Quality (DQ), an active and attractive research area for many years. Owning a huge amount of data could increase the risk of quality degradation and require efficient mechanisms to check data quality and manage quality throw whole process of data lifecycle, from generation to information knowledge extraction. Basics and further reading can be found in [9].

One more interesting area could be Big Data Analytics (BDA), as new way to business analytics then traditional Business Intelligence does. BDA We recommend further reading in [4] and [10].

\section{Conclusion}

The goal of this paper is to conduct a current state-of-art on big data application in telecom domain. Our purpose is research and identify the features of big data technology which can be help for telecom companies in their daily business. Our results indicate that there are at least four areas which need to be considered in order to applicate big data approach for improvement of analytics and business at all. Further research should also consider technical reports and real world use cases in order to better understand telecom needs and new solutions. This review shows that, despite the academic attention that the big data applied in telecoms has attracted to date, there is considerable room for further research on this area.

\section{References}

[1] Azvine, B.; Cui, Z.; Nauck, D.; Majeed, B. (2006). Real Time Business Intelligence for the Adaptive Enterprise. CEC/EEE 2006 Joint Conferences. 2006. 29-29. 10.1109/CEC-EEE.2006.73.

[2] Kocijan, K. (2014). Big Data: how we got the big data and where they lead us, Department of Information Studies, pp. 37-62, Zagreb (in Croatian)

[3] Veda C. S.; Il-Yeol Song (2017). Big data technologies and Management: What conceptual modeling can do, Data \& Knowledge Engineering, Volume 108 (2017), pp. 50-67.

[4] Ting-Penn. L.; Yu-His, L. (2018). Research Landscape of Business Intelligence and Big Data analytics: A bibliometrics study, Expert Systems with Applications, Volume 111(2018), pp. 2-10, ISSN 0957-4174.

[5] Ahmad, A.K., Jafar, A. \& Aljoumaa, K. Customer churn prediction in telecom using machine learning in big data platform. Journal of Big Data 6, article 28 (2019).

[6] Boel, N.; Tomas, O. (2016). Security and Privacy for Big Data: A Systematic Literature Review, 2016 IEEE, International Conference on Big Data (Big Data) 978-1-4673-9005-7/16, IEEE 369

[7] Suleykin, A[lexander] \& Panfilov, P[eter] (2019). Implementing Big Data Processing Workflows Using Open Source Technologies, Proceedings of the 30th DAAAM International Symposium, pp.0394-0404, B. Katalinic (Ed.), Published by DAAAM International, ISBN 978-3-902734-22-8, ISSN 1726-9679, Vienna, Austria

[8] Mohamed, A., Najafabadi, M.K., Wah, Y.B. et al. The state of the art and taxonomy of big data analytics: view from new big data framework. Artif Intell Rev 53, 989-1037 (2020). https://doi.org/10.1007/s10462-019-09685-9

[9] Taleb, I.; Serhani, M.A.; Bouhaddioui, C. et al. (2021). Big data quality framework: a holistic approach to continuous quality management. J Big Data 8, 76 (2021).

[10] Ajah, I.A.; Nweke, H.F. (2019). Big Data and Business Analytics: Trends, Platforms, Success Factors and Applications. Big Data Cogn. Comput. (2019), 3, 32. 